\title{
Three Level Security System using Image Based Authentication
}

\author{
M.Aparna $^{1}$, S.Gopalakrishnan ${ }^{2}$, C.M.Anjusree ${ }^{3}$ \\ Student, Dept. of Computer Technology, Sri Krishna Arts and Science College, Coimbatore, Tamil Nadu, India ${ }^{1,3}$ \\ Assistant Professor, Dept. of Computer Technology, \\ Sri Krishna Arts and Science College, Coimbatore, Tamil Nadu, India ${ }^{2}$
}

\begin{abstract}
Now a days, as information systems are more open to the Internet world, the importance of security for networks is increased. Security is the way of protection to safeguard a nation, persons or person against danger, damage, loss, and crime. Therefore text based passwords are not enough to counter such problems. This demands need for something well secured along with user friendly, Therefore Three level security is an user-friendly software. Where it have increased security by using 3 levels . Level-1 Text based password, Level-2 Image Based Authentication and Level-3 Generating One Time Password.
\end{abstract}

Keywords: OTP, Text Authentication, Image Authentication

\section{INTRODUCTION}

Security plays major role in every network system which we use in our day to day life. In this Three Level Security we have tried to extend the protection by involving a 3-level security approach, involving text primarily based at Level one, Image based Authentication at Level two, and automatic generated one-time password (received through an automatic message to the user) at Level three. In second level the use of distinctive image set within the IBA System Authentication plays a important role in protective resources against unauthorized and smuggled use.

\section{RELATED WORK}

The main Objective of 3-Level Security system is a unique and an esoteric study of using images as password which helps to give extreme secure to the system, thus we are employing 3 levels of security

1. Text Authentication (LEVEL-1)

2. Image Authentication (LEVEL-2)

3. OTP Authentication (LEVEL-3)

\section{TEXT AUTHENTICATION (LEVEL 1)}

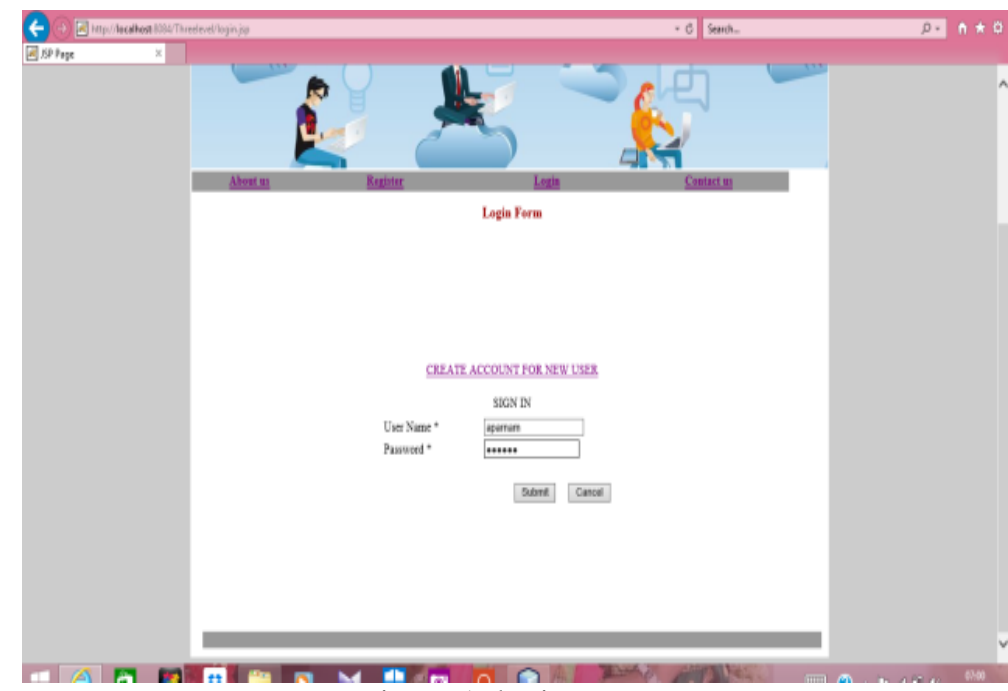

Figure 1: login screen 


\section{International Journal of Advanced Research in Computer and Communication Engineering}

Vol. 7, Issue 11, November 2018

Passwords have been used with computers since the earliest time of computing. This was introduced in 1961. It had a LOGIN command that requests a user password. After typing PASSWORD, the system turns off the printing mechanism, if possible, so that the user may type in his password with privacy. To log in, the user is asked to type the password which already given while creating login. Therefore, security at LEVEL1 is ensured by use of text password they are allowed to have special character which is a usual approach with normal login scheme.

\section{IMAGE AUTHENTICATION (LEVEL 2)}

Image based authentication was developed as an alternative click based graphical password scheme where users select one image from the grid of images, the image is uploaded as soon as a user selects a click point are saved for next login authentication. The system determines the selection of images where the user has to select the same image from the previous selection .If a user enters an incorrect click-point during login, the next click point displayed will not be considered.

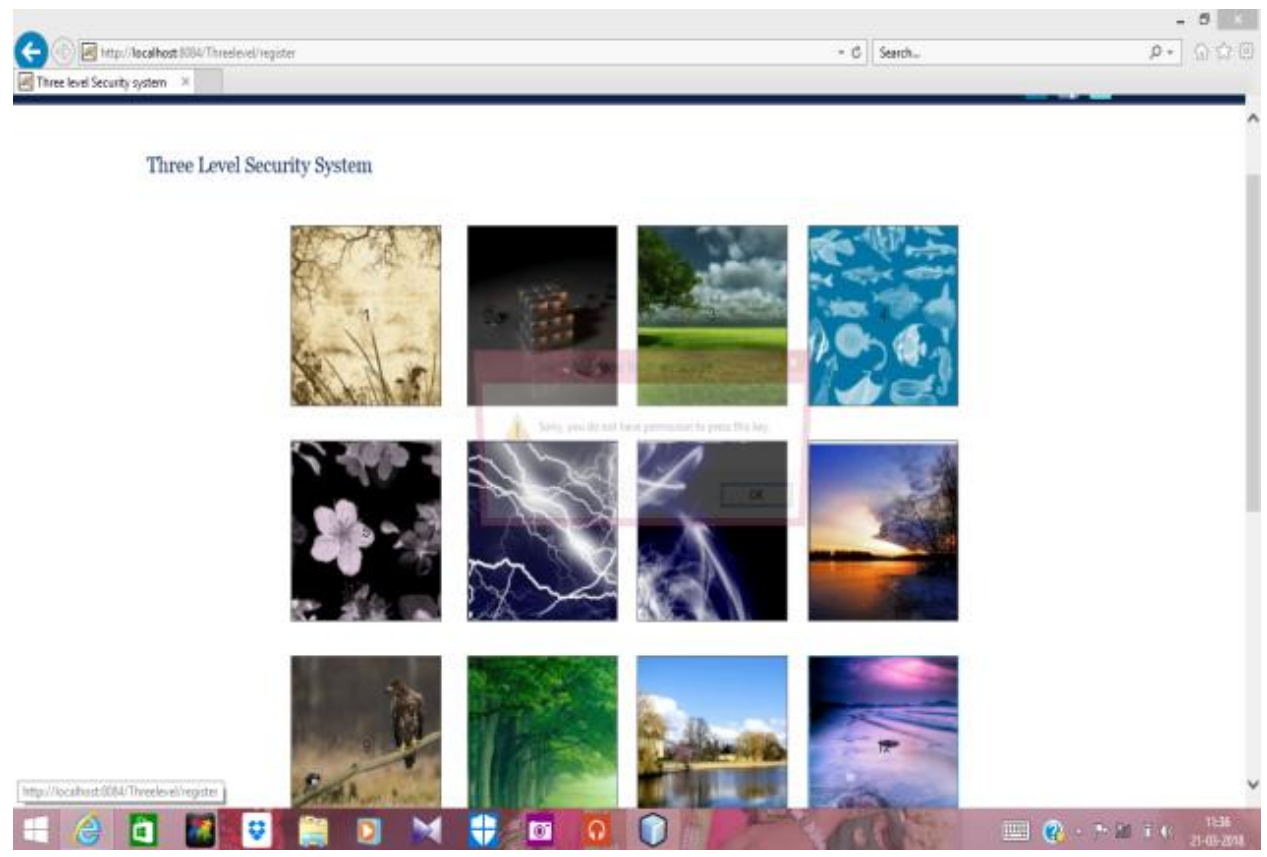

Figure 2 : image selection

\section{OTP AUTHENTICATION (LEVEL 3)}

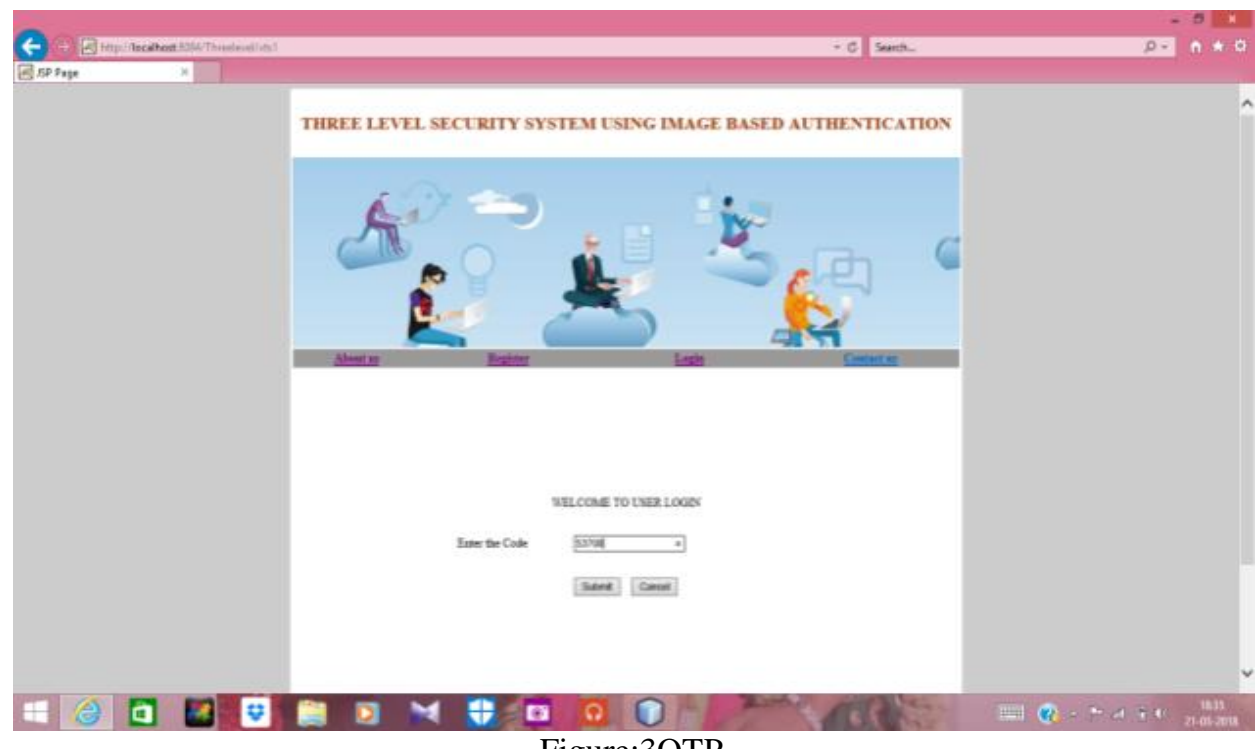

Figure:3OTP 
OTP Authentication LEVEL security has been imposed by generating a onetime random code. This random code or can say the password will be generated each time during the specific login, for the user to login to his account after the successful completion of two authentication processes (security level1- Text password and security level2-Image based authentication). This unique code will be updated in the database of the server. And the user will be informed of this one-time password through an automated phone number. This will definitely help in threatening Brute force attack (can be attempted upon the previous two security levels), as this unique one-time password will be send on user's phone number saved in the database. The user will be allowed to access that one-time password, only upon having access to that phone number.

\section{PROPOSED SYSTEM}

In day to day life, all business, government organizations and other organizations are investing a lot of money and computer memory for the security of information. Online password guessing have been known since the early days of the Internet, there is little academic on prevention techniques.

This project proposes 3 levels of security. During password creation, there is an image user will select three click points or pixel positions within that image. After considering the pixel positions user must re-login and authenticate for the next level of login process i.e., OTP generation sent to the phone number. Therefore this works encouraging users to select Image and difficult Click points to guess. Brute force and dictionary attacks on password - only remote login are now widespread and ever increasing. While preventing such attacks, enabling convenient login for legitimate users is a difficult problem. Automated Turing Tests continue to be an effective, easy - to - deploy approach to identify automated malicious login attempts with reasonable cost to users.

\section{FEATURES}

1. The system is user-friendly and has simple interface.

2. Provides strong security against bot attacks or hackers.

3. Users can set or upload their own images.

4. Protects systems vulnerable to attacks.

\section{CONCLUSION}

In this project "THREE LEVEL SECURITY SYSTEM USING IMAGE BASED AUTHENTICATION". A new type authentication system, which is highly secure has been proposed in this project. This system, is also more user friendly. This system will definitely help Shoulder attack, Tempest attack and Brute-force attack at the client side. Though 3Level Security system is a time consuming approach, it will provide strong security where we need to store and maintain crucial and confidential data secure. Such systems provide a secure channel of communication between the communicating entities. The ease of using \& remembering images as a password also support the scope of these systems.

\section{REFERENCES}

[1]. Security Analysis and Implementation of JUIT-IBA System using Kerberos Protocol, Proceedings of the 7th IEEE International Conference on Computer and Information Science, Oregon, USA, pp. 575-580, 2008.

[2]. Richard E. Newman, Piyush Harsh and Prashant Jayaraman, "Security Analysis of and Proposal for Image Based Authentication," 2005.

[3]. Chiasson, S., R. Biddle, R., and P.C. van Oorschot. A Second Look at the Usability of Click-based Graphical Passwords. ACM SOUPS, 2007.

[4]. Haichang Gao, Zhongjie Ren, Xiuling Chang, Xiyang Liu Uwe Aickelin, A New Graphical Password Scheme Resistant to Shoulder-Surng.

[5]. Z. Zheng, X. Liu, L. Yin, Z. Liu A Hybrid password authentication scheme based on shape and text Journal of Computers, vol.5, no.5 May 2010.

[6]. Chris Ullman and Lucinda Dykes, Beginning Ajax (Programmer to Programmer), Paperback, March $19,2007$. 